(C)2007 IEEE. Personal use of this material is permitted. However, permission to reprint/republish this material for advertising or promotional purposes or for creating new collective works for resale or redistribution to servers or lists, or to reuse any copyrighted component of this work in other works must be obtained from the IEEE. 


\title{
Towards Quantifying the Possible Risk in e-commerce Interactions for RDSS
}

\author{
Omar Hussain, Elizabeth Chang, Farookh Hussain and Tharam Dillon \\ Digital Ecosystems and Business Intelligence Institute \\ Curtin University of Technology, Perth, Australia \\ Omar.Hussain@postgrad.curtin.edu.au, \{Elizabeth.Chang,Farookh.Hussain,Tharam.Dillon\}@cbs.curtin.edu.au
}

\begin{abstract}
By analyzing the possible level of Risk beforehand the initiating agent can make an informed decision of its future course of interaction with an agent. The possible risk in the context of an e-commerce interaction is a multidimensional construct which is the combination of different constituents. In this paper we propose a methodology by which the initiating agent can determine and analyze those constituents in order to ascertain the possible level of risk in interacting with an agent.
\end{abstract}

\section{Introduction}

'Trust' and 'Risk' are substantial concepts in today's world across all disciplines. In today's sophisticated, advanced and developing world the above mentioned two concepts demand importance and hence are important to consider. In the context of e-commerce interactions, these two concepts help the initiating agent of the interaction to analyze, ponder and then to decide its future course in the interaction with any other agent. Considering an instance from our practical daily lives before investing our resources in interacting with an agent over a virtual environment, it is logical for us to think whether we will achieve our desired outcomes in the interaction or not and to fear for the safety of the resources involved in the interaction. Based on our analysis, we decide on our future course of action in interacting with an agent. In other terms the questions that we ask ourselves, or that come in our mind before initiating the interaction, are related to Trust and the Risk that is associated with it. Trust and Risk are the two concepts associated with an interaction, which are important to consider and which complement each other for the initiating agent to make an informed decision of its future course of action with an agent. Due to the importance of these concepts, there have been various conceptualizations of trust and risk according to the discipline in which they are being discussed in [1-8]. The conceptualization of each term depends on how it best expresses its object of analysis in that particular discipline, and hence it varies across disciplines. Subsequently due to the various definitions available for the terms trust and risk, some misconceptualization arises on the relation between them. Trust as it stands out has been linked with risk in many ways and this prompted Mayer et al. to highlight the uncertainty of the relationship between them by stating that "it is unclear whether risk is an antecedent to trust, is trust, or an outcome of trust" [9]. Trust and Risk may be related to each other, but they express different concepts and hence cannot be conceptualized as synonyms to each other. Both of these concepts although complement each other, express different meanings, which in turn cannot be reciprocated to define and analyze the other term. Hence it is incorrect to consider these two terms as synonyms to each other or either to compare and decide as to which one of them is more important for better decision making in an interaction.

As their conceptualization, the way trust and risk in an interaction are quantified and expressed also varies. Trust in an interaction can be expressed in terms of belief or in terms of probability in an interaction, whereas risk in an interaction is best understood when expressed in terms of probabilities under conditions of uncertainty, which tends towards expressing the possible loss in an interaction. As both these concepts are expressed in terms of probability, it may be possible that they both express the probability related of a certain event, but the type of outcome that each concept tries to express are in contrast to each other. While trust refers to the assessed belief or probability of having a desirable outcome performed in an event, risk refers to the assessed probability of NOT having a desirable outcome achieved in that event and the associated loss in not achieving the desired outcomes. In this sense both trust and risk are opposite concepts to each other. By expressing trust and risk as probabilities in an interaction, the magnitude of effect that they have on the expressed outcomes can also be determined according to their probability of 
occurrence. In other terms, trust and risk are two concepts whose evaluation would give two contrast perspectives of occurrence of an event, and their way of analysis and outputs achieved from evaluating them are not just converse of each other, but would provide various insight and would give solutions to the various doubts which rise in the initiating agent's mind before the interaction. In this paper our main aim is to propose a methodology by which the initiating agent of an interaction can utilize it to determine the possible level of risk present in interacting with a probable risk assessed agent, and based on that it can decide better on its future course of interaction with it.

So we term the two agents participating in an interaction as the 'risk assessing agent' and 'risk assessed agent'. The former refers to the one initiating the interaction while the latter refers to the one with whom it interacts with, to achieve its desired outcomes in the interaction. We define the possible risk in an ecommerce interaction as the likelihood that the probable risk assessed agent might not act as expected according to the risk assessing agent's expectations in a given context and at a particular time once the interaction begins, resulting in the loss of $\$$ and the resources involved in the interaction. Hence the possible risk in an interaction is a multidimensional construct which is a combination of the:

- probability of failure of an interaction,

- the consequences of failure of the interaction, and

- the loss of investment probability in the resources of the interaction.

For risk analysis, the risk assessing agent has to determine beforehand the above-mentioned factors in interacting with a probable risk assessed agent. In this paper we propose the methodologies by which the risk assessing agent can determine each of the above mentioned factors to analyze the possible risk in interacting with a probable risk assessed agent. The methodologies are explained in the next sections.

\section{Determining the Probability of Failure in an Interaction}

As mentioned earlier, the risk assessing agent has to determine the probability of failure and the possible consequences of failure to its resources in order to analyze the possible level of risk before initiating its interaction with a risk assessed agent. Hence to quantify and represent semantically the probability of failure of a risk assessed agent, we defined the term 'FailureLevel' and the Failure scale in Hussain et al. [10]. FailureLevel quantifies and semantically expresses the possible level of failure on the failure scale. The Failure scale as shown in Figure 1 represents the different levels of failure, possible in a given period of time. The risk assessing agent determines the FailureLevel in interacting with the probable risk assessed agent beforehand by ascertaining its in-capability to complete the interaction, according to the expectations of its future interaction with it. In other words, the FailureLevel of an interaction is the extent to which the risk assessing agent determines that it might not achieve its desired outcomes in interacting with a probable risk assessed agent.

\begin{tabular}{|c|c|c|c|}
\hline $\begin{array}{l}\text { Semantics of } \\
\text { Failure Level }\end{array}$ & $\begin{array}{c}\text { Probability of } \\
\text { Failure }\end{array}$ & FailureLevels & Star Rating \\
\hline Unknown & . & -1 & Not Displayed \\
\hline Total Failure & $\begin{array}{l}91 \text { - } 100 \% \text { Probability of } \\
\text { Failure }\end{array}$ & 0 & Not Displayed \\
\hline Extremely High & $\begin{array}{c}71-90 \% \text { Probability of } \\
\text { Failure }\end{array}$ & 1 & From 13 to 4 \\
\hline Largely High & $\begin{array}{c}51-70 \% \text { Probability of } \\
\text { Failure }\end{array}$ & 2 & From 4 代 to \\
\hline High & $\begin{array}{c}26-50 \% \text { Probability of } \\
\text { Failure }\end{array}$ & 3 & 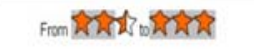 \\
\hline Significantly Low & $\begin{array}{l}11-25 \% \text { Probability of } \\
\text { Failure }\end{array}$ & 4 & From ind \\
\hline Extremely Low & $\begin{array}{l}0-10 \% \text { Probability of } \\
\text { Failure }\end{array}$ & 5 & Fromindutimin \\
\hline
\end{tabular}

Figure 1: The Failure scale

The risk assessing agent communicates its desired outcomes and the resources it will invests to achieve them, to the risk assessed agent before interacting with it, in the expected behavior or the mutually agreed behavior. The expectations or the desired outcomes that the risk assessing agent wants in its interaction with a probable risk assessed agent can be classified at a higher level as the 'Context' of the interaction. It can be decomposed into several detailed aspects known as the 'Criteria', which defines the demand or the set of factors which show specifically what the risk assessing agent wants in its interaction with the risk assessed agent in the particular context. Criteria form the expectations or the desired outcomes of the risk assessing agent. By considering its expectations, the risk assessing agent will accurately determine the probability of failure according to its criteria or desired outcomes.

It may be the case that the possible interaction of the risk assessing agent with the probable risk assessed agent is in the future state of time. Hence, for risk analysis, the risk assessing agent has to determine the FailureLevel in interacting with the probable risk assessed agent in that future state of time. In order to 
achieve that, we propose that the risk assessing agent analyze the FailureLevel in interacting with a probable risk assessed agent in two stages. They are:

1. Pre-interaction start time phase

2. Post-interaction start time phase

'Pre-Interaction start time phase' refers to the period of time before the risk assessing agent starts its interaction with the probable risk assessed agent, whereas 'Post-Interaction start time phase' is that period of time after the risk assessing agent commences and interacts with the probable risk assessed agent. For risk analysis, the risk assessing agent has to determine the FailureLevel in interacting with a probable risk assessed agent in this period of time, that is in the post-interaction start time phase. However, if this phase is in the future state of time, the risk assessing agent can only determine the FailureLevel by using some prediction methods. So to achieve this, we propose that the risk assessing agent should first ascertain the FailureLevel of the probable risk assessed agent according to the specific context and criteria as that of its future interaction in the preinteraction start time phase. Based on those levels, the risk assessing agent can determine its FailureLevel in the post-interaction start time phase. The determined FailureLevel of the probable risk assessed agent in that time phase depicts the probability and level of failure in interacting with it, during the time of the risk assessing agent's possible interaction with it.

It is possible that the risk assessed agent may have varying levels or degree of failure rather than having a concrete level of failure in a given period of time. Also it is mentioned in the literature that, risk is dynamic varying from time to time. As such, the risk assessing agent should take this dynamic nature of risk into consideration while undertaking risk analysis in interacting with a probable risk assessed agent. To incorporate that, we propose the risk assessing agent should divide the total time that it considers to determine the FailureLevel of the probable risk assessed agent, termed as the 'time space', into different non-overlapping parts, termed as 'time slots', and determine the FailureLevel of the risk assessed agent in each of those time slots. By doing so, the risk assessing agent ascertains the correct FailureLevel of the probable risk assessed agent in a time slot, according to its incapability to complete the criterions of its future interaction in that particular time slot, thus considering its dynamic nature while doing risk analysis. The time slots will be spread out either in the pre-interaction or in the post-interaction start time phase. The risk assessing agent has to determine the FailureLevel of the probable risk assessed agent in each time slot according to the time phase in which they fall.

We quantify the level of failure on the failure scale in interacting with a probable risk assessed agent in a given context and at a given time ' $t$ ' which can be either at the current, past or future time with the metric 'FailureLevel'. But for better understanding, we represent the FailureLevel of a probable risk assessed agent according to the time phase in which they are determined and they correspond to. For example, if the FailureLevel for a probable risk assessed agent is determined in the pre-interaction start time phase, then we represent it by the metric 'PFL' which stands for 'Previous FailureLevel'. Similarly, if the FailureLevel for the probable risk assessed agent is determined in the post-interaction start time phase we represent it by 'FFL' which stands for 'Future FailureLevel'.

The risk assessing agent can determine the FailureLevel of a probable risk assessed agent in each of the pre-interaction start time slots of its interaction either by:

a) Considering its past interaction history with the probable risk assessed agent, if it was in that time slot and in the criteria as that of its future interaction with it; or

b) Soliciting recommendations from other agents and determining the in-capability of the probable risk assessed agent to complete the interaction according to the criteria of its future interaction with it.

We have defined a methodology in Hussain et al. [10] by which the risk assessing agent either utilizes its past interaction history or assimilates the recommendations to determine the FailureLevel (PFL) of the probable risk assessed agent on the failure scale, in each time slot of the pre-interaction time start phase. The main points of the proposed methodology are:

- By assimilating the recommendations in a preinteraction time slot, the risk assessing agent determines the FailureLevel of the probable risk assessed agent in each criterion of its future interaction with it.

- It then combines the FailureLevel of each criterion with the significance of that criterion, to determine a crisp FailureLevel of the probable risk assessed agent in that time slot. The significance of each criterion shows the degree to which it influences the successful outcome of the interaction, according to the risk assessing agent.

- The risk assessing agent while assimilating the recommendations gives more weight to those which are from trustworthy recommending agents as compared to those which are from unknown recommending agents. The recommendations from un- 
trustworthy recommending agents are omitted and not considered.

- Also the risk assessing agent gives more weight to those recommendations which are in the recent time slot, to the time spot of its interaction with a probable risk assessed agent, as compared to those which are in the far recent ones, so as to reflect the current status or reputation of that probable risk assessed agent.

Once the risk assessing agent determines the FailureLevel (PFL) of the probable risk assessed agent in each of the pre-interaction time slots, according to the context and criteria of its future interaction with it, then it can determine the FailureLevel (FFL) of the probable risk assessed agent in the post-interaction start time slots. To achieve that, we propose the risk assessing agent should utilise the FailureLevel of the probable risk assessed agent that it had determined according to the specific context and criteria as that of its future interaction in the pre-interaction start time slots and then, based on those levels, it should predict its FailureLevel in the post-interaction time slot. As the determined FailureLevel (PFL) of a probable risk assessed agent in the pre-interaction start time phase is strictly according to the criteria of its future interaction, the future FailureLevel (FFL) determined by utilising these levels too is strictly according those criteria.

We propose that the risk assessing agent while determining the FailureLevel (FFL) of a probable risk assessed agent in a post-interaction time slot, should determine the probability of occurrence of each level of failure within the domain of $(0,5)$ on the failure scale, rather than determining a crisp FailureLevel as it does in the pre-interaction time slots. This is because the future FailureLevel (FFL) of a probable risk assessed agent at time $t+1$ is predicted by considering its FailureLevels from its time space till time t. This might not give an accurate conclusion as compared to the one obtained in the pre-interaction time slots where the risk assessing agent determines the risk assessed agent's FailureLevel by either considering its past interaction history or by assimilating the recommendations. In order address this; we propose that the risk assessing agent in each post-interaction time slot should determine the magnitude of occurrence of each level of failure on the failure scale, rather than determining a crisp FailureLevel, in interacting with the risk assessed agent. By doing so the risk assessing agent would also determine the probability of occurrence of each level of failure on the failure scale in interacting with the probable risk assessed agent in each of the post-interaction time slots according to its criteria.

Our method for determining the future FailureLevel
(FFL) for a probable risk assessed agent at time slot $\mathrm{t}+1$ is by taking its FailureLevels from the beginning of the time space till time $t$ and utilise the Gaussian Distribution to determine the probability of the future FailureLevel (FFL) being any level on the failure scale. As mentioned, the failure scale ranges from $(-1$, 5 ), with -1 denoting unknown level of failure and levels between 0 and 5 denoting varying degree of failure levels. So the future FailureLevel of a probable risk assessed agent in a post-interaction time slot is determined in the domain of $(0,5)$ on the failure scale. Within this domain there are six possible levels of failure on the failure scale.

The number of time slots in the post-interaction time phase of the interaction depends on the risk assessing agent's duration of the interaction and the division of the time space. Hence it may be the case that the time space is of a very long duration for example 1 year or of a very short duration for example 1 day. What ever the case may be, for risk analysis it would be easier and beneficial for the risk assessing agent if it can analyze the possible risk in interacting with a risk assessed agent for the whole period of the post-interaction start time phase in one go, rather than having to analyze it according to each time slot in that time phase of the interaction. Hence to achieve that we propose that the risk assessing agent after determining the FailureLevel of the probable risk assessed agent in each time slot of the post-interaction start time phase, should compute its FailureLevel curve as shown in figure 2. This curve shows the probability of occurrence of different levels of failures possible while interacting with the risk assessed agent in the postinteraction time phase of the interaction. The abscissa of the curve gives the level of failure and the corresponding ordinate or impulse gives the probability of occurrence of that level.

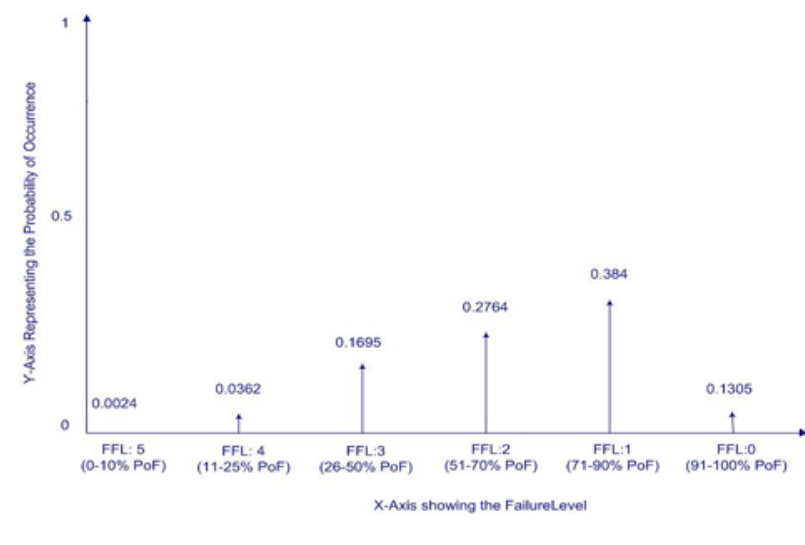

Figure 2: FailureLevel curve of the interaction 
The above curve shows the probability of occurrence of each level of failure on the failure scale as determined by the risk assessing agent in interacting with the risk assessed agent in the post-interaction time phase. Once the FailureLevel curve in interacting with a probable risk assessed agent has been determined, the risk assessing agent should then determine the other two constituents of risk analysis, namely the possible consequences of failure to its resources and the loss of investment probability to its resources in interacting with a probable risk assessed agent. In the next sections we will propose the methodology by which the risk assessing agent can ascertain the above mentioned factors.

\section{Determining the Possible Consequences of Failure in an Interaction}

Like the probability of failure, the possible consequence of failure too is dependent on the context of the interaction, and varies according to the object of analysis in which they have to be determined. So, before analyzing the possible consequences of failure in an interaction, it is first important to ascertain the object of analysis in which they have to be determined, according to the context in which they are being discussed in. In a context, object of analysis identifies the field in which the consequences of failure have to be determined. In this paper we assume that an ecommerce interaction is carried out between the two agents and that the risk assessing agent interacts with the probable risk assessed agent to achieve its demand in exchange of the monetary financial value. Subsequently, in our context, the term 'resources' refers to the financial resources invested by the risk assessing agent in its interaction with the risk assessed agent to achieve its demand or desired outcomes. The risk assessing agent by determining the possible consequences of failure to its resources while interacting with a probable risk assessed agent can ascertain:

- The extra financial resources that it has to invest while interacting with the probable risk assessed, apart from the net resources that it invests according to the expectations,

- The probability to which it will not achieve the full benefit of the net resources that it is investing its resources according to the expectations, while interacting with a probable risk assessed agent.

While determining the possible consequences of failure in interacting with a probable risk assessed agent, the risk assessing agent incorporates and follows the division of time as done by it while determining the probability of failure in interacting with that agent. But the risk assessing agent invests and has its resources at stake in only the post-interaction start time phase of its interaction, and subsequently while determining the possible consequences of failure in interacting with a probable risk assessed agent it has to ascertain it only in that phase of time. As mentioned earlier, the number of time slots in the post-interaction phase depends on the risk assessing agent's classification of the time space of the interaction. If there is more than one time slot in the post-interaction phase, then the net worth of the risk assessing agent's resources at stake in the interaction increases progressively, as the time slots increase according to the total worth invested in each of them. Hence the risk assessing agent should first ascertain the accurate net worth of its resources at stake in the interaction, before determining the possible consequences of failure in those. This property is important to consider as it is possible that the risk assessing agent might not have the full amount of its resources at stake, as decided in the expectations throughout the duration of the post-interaction start time phase. So we propose that before ascertaining the possible consequences of failure, the risk assessing agent should first determine the accurate net worth of its monitory financial value at stake from its resources invested, during the post-interaction start time phase of its interaction. The accurate worth of the risk assessing agent's resources at stake in the interaction depends on the nature of how it invests those resources in it. It is possible that the risk assessing agent might invest the total threshold of its resources as decided in the expectations at once at the beginning of the postinteraction start time phase, or it might invest it progressively in each time slot of its post-interaction phase. In each scenario, the amount of the risk assessing agent's monitory value invested and at stake throughout the duration of the post-interaction phase varies, subsequently scaling the possible consequences of failure along with it. So to take this variation into consideration, we propose that the risk assessing agent while ascertaining the possible consequences of failure in an interaction should determine it according to the net worth of its resources that it has at stake.

In order to get a better understanding of the reason to ascertain the accurate net worth of the risk assessing agent's resources at stake before determining the possible consequences of failure in an interaction, let us consider an example of risk assessing agent ' $A$ ' wanting to interact with a probable risk assessed agent ' $\mathrm{B}$ ' for a period of 1 year. Let us suppose that the risk assessing agent ' $A$ ' forms the time space of its interaction, and divides it such that there are 10 time 
slots in the post-interaction start time phase (t1-t10). Further the risk assessing agent ' $A$ ' decides with the probable risk assessed agent in its expectations to invest $\$ 20,000$ in the interaction, to achieve its desired outcomes. But before determining the possible consequences of failure in an interaction, the risk assessing agent has to first ascertain the accurate net worth of its resources at stake during the postinteraction start time phase of its interaction, which depends on the nature of investment of its resources in the interaction. Two possibilities arise in the nature of the risk assessing agent investing its resources in the interaction.

- Either it is possible that the risk assessing agent invests the maximum threshold of its resources at once in the beginning of the post-interaction start time phase, or

- It may invest it progressively or in stepwise way, in each time slot of the post-interaction start time phase.

For example, it is possible that the risk assessing agent invests $\$ 20,000$; the total net worth of its resources as decided in the expectation in the first time slot of the post-interaction phase, or it may invest the total worth of its resources progressively, that is say in the order of $\$ 2,000 ; \$ 3,000 ; \$ 6,000 ; \$ 5,000$ and $\$ 4,000$ in time slots $1,3,4,6$ and 9 of the post-interaction time phase respectively, to gradually make the total worth of the interaction $\$ 20,000$. In both cases, the probability of an amount invested from its resources throughout the duration of the post-interaction phase is different. The possible consequence of failure which the risk assessing agent has to determine while interacting with a probable risk assessed agent should be according to the resources that it has at stake in the interaction. So for the risk assessing agent to determine accurately the net worth of its resources at stake in an interaction, we propose the calculation of an Amount Invested Curve $(A I C)$. This curve gives the probability of an amount invested and at stake from the net resources as decided in the expectations, throughout the duration of the post-interaction start time phase to achieve the desired outcomes of the risk assessing agent. The essence of determining such a curve is for the risk assessing agent to ascertain the consequences of failure in the interaction accurately according to net worth of its resources at stake. Figure 3 shows the AIC of the interaction when the resources of the risk assessing agent are invested progressively.

To ascertain and quantify the degree of loss in the investment of the risk assessing agent, we propose the calculation of the Factual Amount Invested Curve (FAIC). The factual amount invested curve shows the

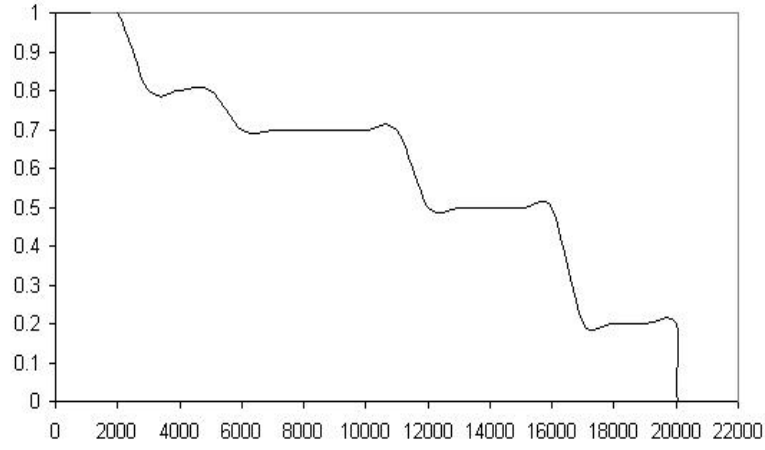

Figure 3: Amount invested curve for the interaction

required probability of an amount to be kept at stake by the risk assessing agent, throughout the duration of the interaction to achieve its desired outcomes, by taking into consideration the FailureLevel of the risk assessed agent and the probability of that amount it was initially investing. Hence, the factual amount invested curve (FAIC), which shows the increased probability of an amount that the risk assessing agent needs to invests in an interaction, is an extension of the amount invested curve (AIC). The AIC shows the actual probability of an amount invested and at stake from the resources of the risk assessing agent throughout the duration of the interaction, according to the expected or mutually agreed behavior, whereas the FAIC shows the required probability of the risk assessing agent to invest that amount throughout the duration of the interaction by considering the FailureLevel of the risk assessed agent.

To obtain the FAIC of an interaction, the AIC should be convolved with the FailureLevel curve of the risk assessed agent. The convolution of the two graphs can be done either by the conventional method or by using the cumulant method. In the conventional method each point on the AIC has to be convolved with the FailureLevel curve to obtain the FAIC of the interaction, whereas in the cumulant method the convolution of the independent random variables can be expressed as a sum of their individual cumulants, which can then be used to model the output FAIC curve by using either Gram-Charlier series expansion or Beta distribution. The output of the convolution, which is the FAIC, is an inflated curve as compared to the AIC as it shows the increased probability of an amount that the risk assessing agent needs to invest in the interaction as compared to what was decided earlier.

To represent with an example the FAIC of an interaction, we convolve the FailureLevel curve of the risk assessed agent shown in figure 2 with the Amount Invested Curve shown in figure 3 to determine the 
Factual Amount Invested Curve of the interaction shown in figure 4. As can be seen from the curves, the FAIC of the interaction is inflated as compared to the AIC as it represents the increased probability of an amount to be at stake in an interaction.

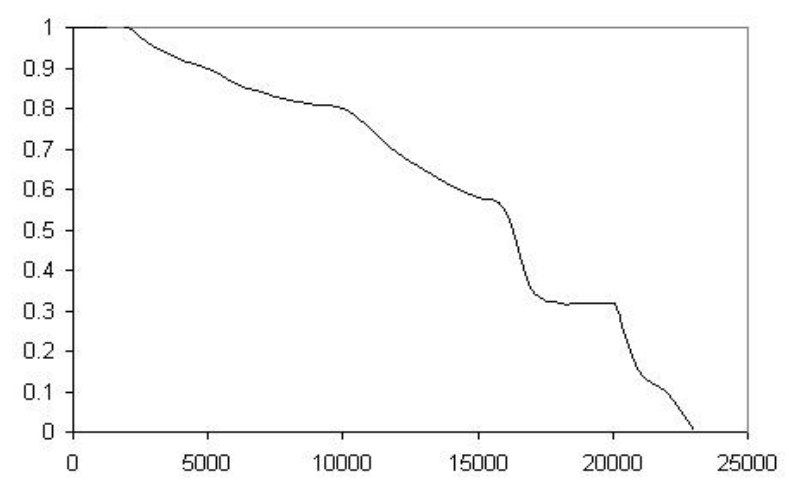

Figure 4: Factual Amount invested curve for the interaction

As discussed earlier, the possible consequences of failure in an interaction are referred to the extra financial resources that the risk assessing agent has to invest in the interaction while interacting with the probable risk assessed agent. The risk assessing agent can ascertain the extra financial resources needed in the interaction by utilizing the Factual Amount Invested Curve and then determining the point on it which represents its maximum capacity to invest the resources in interacting with a probable risk assessed agent to achieve its expectations. In most scenarios, the maximum investment capacity of the risk assessing agent in interacting with a probable risk assessed agent is decided in the expectations, and shown in the AIC. But in certain cases it is possible that apart from the resources that it had decided with the probable risk assessed agent in the expectations, the risk assessing agent might have reserve resources that it can invest, to achieve its desired outcomes in interacting with that agent. In such cases, the maximum capacity which the risk assessing agent can invest is the sum of the resources decided in the expectations and its reserve resources. So to ascertain the possible consequences of failure in an interaction, the risk assessing agent has to first ascertain the maximum capacity to which it can invest its resources in interacting with a probable risk assessed agent. By determining its maximum capacity of investment on the FAIC, the risk assessing agent can ascertain the probability to which it will not achieve the full benefit of its resources in interacting with the probable risk assessed agent. We term the probability to which the risk assessing agent will not achieve the full benefit of its resources in interacting with a probable risk assessed agent as the Loss of Investment Probability (LOIP) in the interaction.

The Loss of Investment probability (LOIP) of an interaction can be determined by ascertaining the probability to which the risk assessing agent will not achieve the full benefit of its resources invested, due to the probable risk assessed agent not completing its desired outcomes in those invested resources. Hence, LOIP index of an interaction is simply the ordinate on the FAIC, at the end of maximum investment capacity of the risk assessing agent. Because by definition of FAIC, this ordinate is the probability of the corresponding amount needed to be at stake in the interaction, but this amount will not invested by the risk assessed agent as it is more than what was initially agreed upon.

Extending the previous discussion, if the maximum investment capacity of the risk assessing agent is $\$ 20,000$ as decided in the expectations then the Loss of investment probability (LOIP) of its resources in the interaction can be determined by:

$$
\mathrm{LOIP}=\mathrm{FAIC}(\mathrm{w})
$$

where, $\mathrm{w}$ is the maximum investment capacity of the risk assessing agent, and

FAIC $(\mathrm{w})=$ Factual amount invested curve after investing the total resources of the interaction.

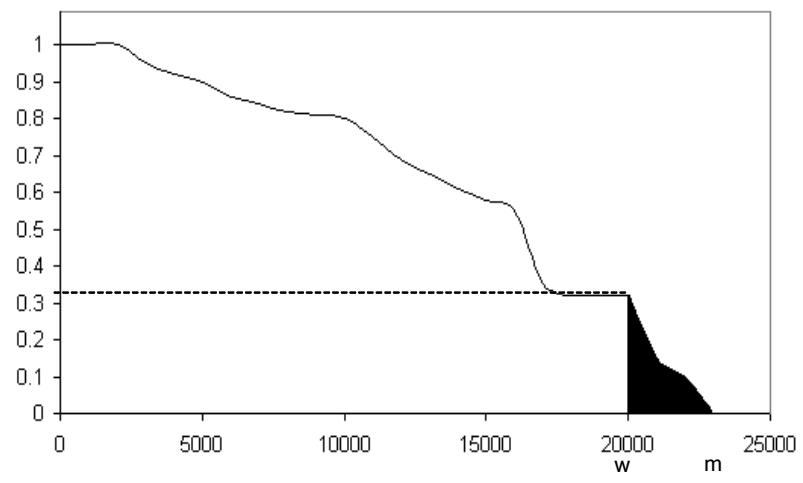

Figure 5: Showing the extra financial resources to be invested and the LOIP in the Interaction

The possible consequences of failure of the interaction is the level of additional resources which the risk assessing agent has to invest in the interaction apart from its maximum capacity of investment, due to the FailureLevel of the probable risk assessed agent in committing to its desired outcomes of the interaction in the resources as promised initially. In other terms, the additional resources needed to be invested are the accumulated resources which theoretically are beyond the maximum capacity to which the risk assessing agent can invest in the interaction. Hence the 
additional resources which the risk assessing agent might have to invest in an interaction is proportional to the area under the FAIC beyond the point w, which represents its maximum capacity to which it can invest its resources as shown in figure 5. The additional resources to be invested in the interaction can be determined by: Additional Resources to be Invested $=\int_{w}^{m} \mathrm{FAIC}(\mathrm{x}) \mathrm{dx}$ where: $\mathrm{w}=$ maximum capacity of the risk assessing agent in investing its resources,

$\mathrm{m}=$ the point where the FAIC ends.

The above equation gives the area under the FAIC from the point of maximum investment capacity of the risk assessing agent. In other terms this area represents the additional resources which the risk assessing agent has to invest in the interaction apart from its maximum investment capacity, to achieve its desired outcomes in interacting with a probable risk assessed agent, due to the FailureLevel. The possible consequences of failure in the interaction can then be determined by comparing the level of additional resources that the risk assessing agent has to invest in interacting with the probable risk assessed agent, with what it can invest in the interaction according to its maximum capacity. To achieve that the risk assessing agent has to first ascertain the area under the FAIC till its maximum investment capacity. We term the area of under the Factual Amount Invested Curve till its maximum capacity of investment as the maximum investment capacity of the risk assessing agent. This can be determined by:

$$
\int_{0}^{w} \operatorname{FAIC}(\mathrm{x}) \mathrm{dx}
$$

where: $\mathrm{w}=$ represents the maximum investment capacity of the risk assessing agent.

The risk assessing agent can determine the possible consequences of failure in the interaction by comparing the area of the curve which shows the additional resources that it has to invest, with the area of the curve which shows the actual resources that it will invest in the interaction. Hence the possible level of consequence of failure in the interaction is determined by:

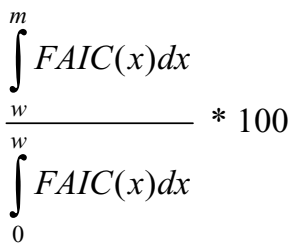

The level of possible consequence of failure in an interaction will be proportional to the area of the curve under the FAIC, after the point $w$. The greater the area of the FAIC curve after point $w$, higher will be the possible of consequence of failure and vice versa.

Once the risk assessing agent determines the probability of failure, the possible consequences of failure to its resources and the loss of investment probability to its resources while interacting with that agent then it can combine them to ascertain the possible risk in interacting with that agent.

\section{Conclusion}

In this paper we proposed a methodology by which the risk assessing agent of the interaction can determine the probability of failure, the possible consequences of failure and the loss of its investment probability in the interaction, by which it can analyze the possible level of risk present in interacting with a probable risk assessed agent in the context of an ecommerce interaction. By combining these constituents the risk assessing agent can make an informed risk based decision of its future course of interaction with that agent.

\section{References}

[1] J. Carter and A.A. Ghorbani, "Towards a formalization of Trust" Web Intelligence and Agent Systems, Vol. 2, No. 3, 2004, pp. 167-183.

[2] Chang, E., T. Dillon, and F. K. Hussain, Trust and Reputation for ServiceOriented Environments: Technologies for Building Business Intelligence and Consumer Confidence, 1st edition, John Wiley and Sons Ltd, England, 2006.

[3] Y. Wang; Fu-ren Lin, "Trust and Risk Evaluation of Transactions with Different Amounts in Peer-to-Peer E-commerce Environments", Proceedings of the IEEE International Conference on e-Business Engineering (ICEBE), October 2006, pp. 102-109.

[4] D. Gefen, V.S. Rao and N. Tractinsky, "The conceptualization of trust and their relationship in electronic commerce: The need for clarification", Proceedings of the 36th Hawaii International Conference on System Sciences, Hawaii, 2003, pp 192-201.

[5] D.M. Rousseau, S.B. Sitkin, R.S. Burt and C. Camerer, "Not so different after all: A cross-discipline view of trust", Academy of Management Review, vol. 23, no. 3, 1998, pp. 391-404.

[6] C. Cheung and M.K.O. Lee, "Trust in Internet shopping: A proposed model and measurement instrument", Proceedings of the $6^{\text {th }}$ Americans Conference on Information Systems, 2000, pp 681-689.

[7] N. Luhmann, 'Familiarity, confidence, trust: Problems and alternatives', Making and Breaking Cooperative Relations, Basil Blackwell, New York, USA, 1988, pp 94-107.

[8] J.G. March and Z. Shapira, "Managerial perspective on risk and risk taking", Management Science, vol. 33, no. 11, 1987, pp. 1404-1418.

[9] R.C. Mayer, J.H. Davis and F.D. Schoorman, "An interactive model of organizational trust", Academy of Management Review, vol. 20, no. 3, 1995, pp.709-734

[10] O. K. Hussain, E. Chang, F. K. Hussain and T. S. Dillon, “A methodology to quantify failure for risk-based decision support system in Digital Business Ecosystems", Data \& Knowledge Engineering, Elsevier Science (Article in Press). 